\title{
VORTEX DYNAMICS IN TURBULENCE
}

\section{I. Pullin}

Graduate Aeronautical Laboratories 105-50, California Institute of Technology, Pasadena, California 91125

\section{P. G. Saffman}

Applied Mathematics 217-50, California Institute of Technology, Pasadena, California 91125

KEY WORDS: decay of turbulence, high Reynolds number

\section{ABSTRACT}

We survey attempts to construct vortex models of the inertial-range and fine-scale range of high Reynolds number turbulence. An emphasis is placed on models capable of quantitative predictions or postdictions.

"I have no satisfaction in formulas unless I feel their numerical magnitude." — Lord Kelvin

\section{Introduction}

In this review, we concentrate on the role that vortex dynamics has played in the understanding of high Reynolds number turbulence. Batchelor (1953, p. 81) remarked that "two mathematical contributions (possibly unattainable) would greatly increase our ability to analyse the decay of turbulence. One is the deduction of a general solution of the Navier-Stokes equation in closed form, for an arbitrary initial spatial distribution of velocity ...."1 Experience with Burgers' equation

$$
\frac{\partial u}{\partial t}+u \frac{\partial u}{\partial x}=v \frac{\partial^{2} u}{\partial x^{2}},
$$

for which the Cole-Hopf closed form solution for arbitrary initial data exists, suggests that reaching this goal would not be particularly helpful. On the other hand, it can be argued that the intense study of vortex dynamics and vortex

\footnotetext{
${ }^{1}$ The other, the formulation of a single dynamical equation describing the time variation of the complete probability distribution of the velocity field, is outside the scope of the present discussion.
} 
interactions in viscous and inviscid incompressible fluids that has taken place in the last few decades has produced sufficient increase in our understanding and store of approximate and/or model solutions of the Navier-Stokes equations in their vorticity form that we can construct physical models of turbulent structure and evolution to be compared with experimental and numerical simulation.

For the purposes of this article, a clear definition or understanding is necessary of what is meant by turbulence and vortex dynamics. Here, we restrict our definition of turbulence to the following: a three-dimensional random motion that is unpredictable in detail, of an incompressible, uniform density, viscous fluid that satisfies the incompressible Navier-Stokes equations and that is either the decay of an initial velocity distribution or is driven by a deterministic or random force field. Turbulence in the real world is almost invariably three dimensional and can be studied by experiment; two-dimensional turbulence, excluded from the present review, is a consequence of the construction of large computers. Nevertheless it is an important research field and deserves its own review.

Because the flow is determined entirely by the distribution of vorticity, it is a truism to say that the evolution of a turbulent velocity distribution is a problem of vortex dynamics. We need, therefore, to be selective in the definition of vortex dynamics. Examples of vortex dynamics not considered are Taylor's $(1915,1932,1935,1937)$ vorticity transfer theory for calculation of velocity profiles in shear flow turbulence and the Taylor-Green problem (Taylor \& Green 1937) that demonstrates the growth of vorticity by the stretching of vortex lines, which is the basic mechanism of energy dissipation. Kolmogorov flows (see Meshalkin \& Sinai 1961, Arnold 1991) are equilibrium distributions of vorticity maintained against dissipation by an external force and are not discussed here, such as the morphological approach to vortex mechanics (e.g. Melander \& Hussain 1994), on the grounds that though they are of potential importance, such studies have not yet made hard contact with turbulence data.

To avoid confusion over the definition of a vortex, which appears to be a matter of controversy (Jeong \& Hussain 1995), we follow Saffman \& Baker (1979) (see also Saffman 1992), and we define a vortex to be a compact region of vorticity, possibly unbounded in one direction, surrounded by irrotational fluid. Strictly speaking, the viscosity has to vanish for this definition to make sense, but we suppose that the viscosity is very small and allow transcendentally small vorticity outside the vortex. Vortex dynamics then refers to the motion of such vortices under the influence of other vortices and/or their own self-induced velocity and possible external irrotational strain. Classic examples of vortices are vortex filaments, when the vorticity is confined to the interior of a tube of small cross section, and vortex blobs such as Hill's spherical vortex.

The question to be addressed is the understanding of turbulence that is gained from the study of vortex dynamics. "Understanding" is a subjective term, and it 
is better to think in terms of quantitative prediction. Thus our test of relevance to the study of turbulence is heavily influenced by the ability of a particular model or calculation to make direct contact with data either from experiment or from direct numerical simulation in some definite numerical form, a philosophy that is expressed succinctly in the quotation above from Kelvin, which we have borrowed from Jeffreys \& Jeffreys (1950). In this view, a successful or partially successful calculation will typically begin from a preferably derived, but more usually prescribed, model vorticity distribution and, after invoking vorticity dynamics and kinematics (which includes statistical kinematics), will proceed to a definite prediction/postdiction of some measurable quantity. The number of quantities calculated should be greater than the number of quantities prescribed in the model vorticity distribution. This filter will exclude many interesting studies of the dynamics of vortices that may contribute to "understanding" turbulence but that have yet to be developed to the point of explicit calculation of turbulence properties: for example, the studies of nonaxisymmetric Burgers' vortices by Robinson \& Saffman (1984), Buntine \& Pullin (1989), and Moffatt et al (1994). Vortex-based numerical simulations have made significant recent progress toward the calculation of complex flows that may be partially or wholly turbulent. This topic has been extensively reviewed (Chorin 1980, Leonard 1980, 1985, Aref 1983, Meiburg 1995) and we do not revisit this work except to discuss several filament-based simulations of inertial-range turbulence.

The general theme of vortex dynamics in turbulence may be expressed in the hypothesis that certain ranges of the broad spectrum of turbulence scales can be described as comprising ensembles of more or less coherent laminar vortex structures that either evolve internally or interact dynamically. We are often interested in the limit of infinite Reynolds number, where in the absence of boundaries, the inviscid Euler equations are assumed to properly describe the flow dynamics. We consider in Section 2 some heuristic models, followed in Section 3 by discussion of what can be learned from a study of two exact solutions of vortex dynamics, one for the Euler equations and the other for the Navier-Stokes equations. In Section 4, we describe some kinematic vortex models that contain no vortex dynamics but that make some interesting predictions. Numerical computation of filament models is considered in Section 5, followed in Section 6 by an account of the stretched-spiral vortex and its application to calculation of several fine-scale turbulent properties. Analysis of possible singularity formation in Euler vortex dynamics is described briefly in Section 7.

\section{Rough Models}

The discovery by Batchelor \& Townsend (1949) and prediction by Landau (see Landau \& Lifshitz 1959, p. 126) of intermittency in high Reynolds number 
turbulent flow has led to extensive research into the existence of "organized eddy structures" or "coherent structures" (e.g. see Townsend 1987). One important issue is the shape or geometry of the coherent eddies, of which the two principal candidates are tubes or sheets of vorticity. The work of Kuo \& Corrsin (1972) presented convincing evidence that the fine-scale structure was more likely to consist of tubes than other possibilities. This has tended to be confirmed by subsequent work, especially the recent direct numerical simulation calculations of the last five years. In addition to the fine-scale tubular structures, the experiment of Brown \& Roshko (1974) demonstrated existence of large-scale tube-like structures in the turbulent mixing layer. Ideas of this kind suggested simple and heuristic physical models of both the fine-scale and the large-scale character of the flow. For example, Tennekes (1968) modeled turbulence as vortex tubes of diameter $\eta$ (the Kolmogorov length) stretched by eddies of scale $\lambda$ (the Taylor microscale), extending an idea of Corrsin (1962) in which the fine-scale structure was modeled by vortex sheets. The third-order skewness, $S$, and kurtosis or fourth-order flatness factor, $K$, of $(\partial u / \partial x)$-type signals were calculated. The skewness was found to be independent of $R_{\lambda}$, the Reynolds number based on the Taylor microscale, and $K$ was proportional to $R_{\lambda}$.

Saffman (1970) developed a heuristic vortex sheet model and made predictions about the way in which flatness and skewness factors of arbitrary order depended upon the Reynolds number. Estimates of structure functions were also made. The results for the third- and fourth-order factors agree with those of Tennekes. However, agreement with experiments is not particularly good (Frenkiel \& Klebanoff 1971), and the consensus is that this type of rough model is too simple for the fine-scale structure and does not contain sufficient dynamics.

\section{Exact Solutions}

In a pioneering paper, Synge \& Lin (1943) examined the consequences of assuming that isotropic turbulence could be modeled as a random superposition of Hill's spherical vortices. Hill's spherical vortex (see e.g. Saffman 1992, section 2.1 ) is a sphere containing vorticity in which the vortex lines are circles about the direction of propagation and the magnitude of the vorticity is proportional to the radius of the circle. In cylindrical polar coordinates $(s, \theta, z), \omega_{\theta}=\mathcal{A} s$, for $s^{2}+z^{2} \leq a^{2}$. Here, $a$ is the radius of the sphere, and $\mathcal{A}$ is related to the propagation speed $U$ of the vortex by $\mathcal{A}=15 U / 2 a^{2}$. Synge $\&$ Lin first fix two points in laboratory axes, say $A, B$ separated by a distance $r$, and then calculate the contribution to the two-point one-time longitudinal velocity correlation function $u^{2} f(r)$, where $u$ is the root-mean-square (rms) velocity component, produced by the velocity field of Hill's spherical vortex with given position and orientation relative to $A, B$. The calculation is based on a one-vortex probability 
density function of vortex position and orientation relative to the fixed points. There is therefore no dynamical or statistical correlation between vortices in different locations. An ensemble average is then performed by integrating over all possible vortex positions, assumed to be distributed uniformly over all space, and all orientations of the vortex axes, assumed to be uniformly distributed on the unit sphere. This gives statistical isotropy. Synge \& Lin calculated $f(r)$ over its entire range, finding in particular that

$$
f(r) \sim \frac{7}{80}\left(\frac{2 a}{r}\right)^{3} \quad \text { as } r \rightarrow \infty .
$$

Equation 2 appears not to have been recognized to be inconsistent with the dynamical theorem on the existence of the Loitsiansky invariant, which says that

$$
u^{2} \int_{0}^{\infty} r^{4} f(r) d r=\text { constant. }
$$

The physical model implies that the Loitsiansky invariant does not exist. This constitutes a success of physical modeling, as subsequent work by Saffman (1967) showed the result to be essentially correct; the Loitsiansky invariant does not exist.

Hill's spherical vortex is inviscid and steady. It is perhaps best viewed as a viable structure for the modeling of the large energy-containing eddies because, owing to the absence of small-scale internal structure or of a continuous dissipation mechanism, its use in calculating turbulence properties of the inertial and dissipation ranges is limited.

A simple exact solution of the Navier-Stokes equations is given by the steady axisymmetric Burgers' (1948) vortex

$$
\omega_{0}(s)=\frac{a \Gamma_{0}}{4 \pi v} \exp \left(-\frac{s^{2} a}{4 v}\right),
$$

where $s$ is the radial distance to the vortex center, which describes a steady axisymmetric distribution of vorticity $\omega(s)$ embedded in an axially symmetric and uniform strain field whose principal extensional rate of strain $a$ is aligned with the vorticity. The total circulation is $\Gamma_{0}$ (see also Saffman 1992, section 13.3). Equation 4 and a similar solution for a stretched shear layer represent a dynamical balance between radial viscous diffusion of vorticity and vortex stretching by the imposed strain. These are attractive candidates for modeling the fine scales of turbulence because they encapsulate the stretching of vortex lines by the local strain and the dissipation of enstrophy by viscosity. Arguments for these mechanisms based on the von Kármań-Howarth (von Kármań $\&$ Howarth 1938) equation at zero separation indicate that they are operative 
in either decaying or forced turbulence at large Reynolds numbers. Tube-like structures have been reported in many numerical simulations, for example by Kerr (1985, 1990), Ashurst et al (1978), Vincent \& Meneguzzi (1991), Jiménez et al (1993), and Porter et al (1994). In these papers the term "tube" appears to mean a locally compact nearly axisymmetric distribution of vorticity. The most recognizable tubes seem to constitute the regions of the most intense vorticity (Jiménez et al 1993), but it is not known presently what proportion of the total vorticity lies either within tube-like structures or within the dominant velocity field of identifiable tubes.

As is clear from Section 2, the use of tubes and sheets predates much of this debate. Townsend (1951) used random ensembles of axisymmetric and plane Burgers' vortices to calculate velocity spectra in the dissipation range for isotropic turbulence. His paper is difficult to follow because he tries to calculate the one-dimensional spectrum directly from the ensemble, but it appears to be the first attempt to obtain a quantitative result at large wavenumbers from an assumed vorticity structure. For the axisymmetric Burgers' vortex, the singlevortex shell-summed energy spectrum is

$$
E_{0}(k)=\frac{N \tau_{2} \Gamma_{0}^{2}}{4 \pi k} \exp \left(-\frac{2 k^{2} v}{a}\right),
$$

where $k$ is the modulus of the wavenumber and $N$ and $\tau_{2}$ are model parameters to be defined subsequently. In contrast, the spectrum of the plane Burgers' layer is proportional to $k^{-2} \exp \left(-2 k^{2} v / a\right)$ times a weakly varying function of $k$ (see Pullin \& Saffman 1994, appendix A). Thus the Kolmogorov exponent $-5 / 3$ lies between the sheet-like and tube-like values, which suggests immediately that vorticity distributions that have both a sheet-like and a tube-like character might have interesting predictive properties in the inertial and dissipation ranges. We remark that the Townsend-Burgers' vortex dissipation range shows a Gaussian rolloff rather than the exponential one seen in both direct numerical simulation and experiment (Kraichnan 1959, Kida \& Murakami 1987, Kerr 1990, Saddoughi \& Veeravilli 1994). Further, volume-filling ensembles of Townsend-Burgers' vortices give poor results for the higher order moments of the longitudinal velocity derivatives for isotropic turbulence (Saffman \& Pullin 1996).

\section{Kinematic Models}

There have been attempts to construct vortex-based kinematic models based on a more extended vorticity structure than those of the models described so far. No vortex-dynamical equations are used, but some contact with turbulence measurements is made. Chorin $(1986,1988,1992)$ has developed a vortexlattice model of the inertial-range scales. There are several versions of the 
model, and here we describe that given by Chorin (1986). See Chorin (1988, 1992) for further developments.

The vortex structure is represented by lines composed of contiguous elements, each positioned on the orthogonal generators of a three-dimensional rectangular lattice. There may be several such "vortices" on the lattice, each with constant circulation, and these are not allowed to intersect. An energy functional is defined that models in a discrete way the energy of a continuous vorticity distribution:

$$
T=\frac{1}{8 \pi} \int d \mathbf{x} \int \frac{\omega \cdot \omega}{\left|\mathbf{x}-\mathbf{x}^{\prime}\right|} d \mathbf{x}^{\prime},
$$

where $\boldsymbol{\omega}(\mathbf{x})$ is the vorticity. In this sense the model has kinematic content. The equations of motion are not used explicitly, but instead rules are formulated that mimic the plausible interaction between vortex elements. Thus, at any time instant, an element, as part of a particular vortex, is stretched by deforming its shape from the straight line joining lattice nodes to a U-shape that contains one edge parallel to and two edges normal to the original element. The vortex element thus increases its length and vorticity by a factor of 3 while reducing its radius by $3^{-1 / 2}$. This represents vorticity stretching on the lattice. Deformations are not allowed to produce intersections, and the deformation rules are governed by constraints on the variation of the energy functional conditioned by entropy considerations so as to produce statistical equilibrium.

Vortex folding is observed in numerical simulations. The energy spectrum is obtained by first estimating the Hausdorf dimension (which has yet to be measured from experiment or from direct numerical simulation) of the lattice vortex structure, followed by the use of scaling and dimensional arguments. An exponent $\mu=1+17 / 24$ is found in the power-law form of the shell-summed inertial-range energy spectrum. The model seems to be predicated on the idea that fine details of the dynamical interactions between vortices and internal to the vortex structures are of less importance than is a fair representatation of the main qualitative physical effects of stretching on the increase in energy of the individual elements, which represents the cascade. In this sense, the model may be interpreted as a "statistical cartoon" of the inertial range. There are few equations and much use of scaling arguments, which is consistent with the explicit philosophy of a halfway house between the phenomenology- and dynamics-based models.

A simple kinematic model of turbulence is provided by the fields induced by a distribution of large numbers of discrete vortex elements. Min et al (1996) discuss the probability density functions for the velocity and for the velocity difference for singular and blob-like vortex elements in two and three dimensions. In the latter case, they argue for a $|\delta u|^{-5 / 2}$ decay in the tail of the 
probability density distribution of the two-point velocity difference $|\delta u|$, when the separation between the two points is large.

The models discussed previously consider isotropic turbulence. A vortexbased description of energy containing scales in a real turbulent flow should introduce inhomogeneity and account for the boundary conditions that create the vorticity. Townsend's (1976) "attached eddy" hypothesis for the turbulent boundary layer has been developed in a quantitative way by Perry \& Chong (1982) and Perry \& Marušić (1995). They suppose that the vortex structure of the log region of a turbulent boundary layer is composed of slender vortex rods with a $\Lambda$-like shape. A two-parameter model of the turbulent mean velocity profile (Coles 1956) is assumed and used in the mean momentum equation to infer the distribution of turbulent shear stress $-\overline{u_{1} u_{3}}$ produced by products of velocity fluctuations $u_{1}$ in the streamwise direction and $u_{3}$ normal to the wall. It is further assumed that the turbulent boundary layer comprises distributions of $\Lambda$-like eddies with given probability-density functions (pdfs) of density per unit volume and scale in relation to the boundary-layer thickness. For a given eddy shape, the mean velocity profile and the $\overline{u_{i} u_{j}}$ profiles are calculated analytically from the vortex kinematics of the rods, and parameters in the pdfs are fixed by fitting the $\overline{u_{1} u_{3}}$ results to those obtained from the mean momentum equation. The model is tested by comparing the profiles of the remaining components of $\overline{u_{i} u_{j}}$ to the experiments of Skåre \& Krogstad (1994) with fair results. The model also produces predictions, which include various one-dimensional spectra of the $\overline{u_{i} u_{j}}$, in excess of parameters. The significance of the work lies in the attempt to construct a quantitative vortex morphology of a classical turbulent flow, namely the highly nonhomogeneous, constant pressure-gradient turbulent boundary layer.

\section{Vortex Filaments}

The possibility that the dynamics of vortex filaments could help to understand the large-scale properties of inhomogeneous turbulent flows was examined by Robinson \& Saffman (1982). They examined the stability of three classical steady vortex configurations of filaments to three-dimensional disturbances in an incompressible inviscid fluid in the limit of small vortex cross-sectional area and long axial disturbance wavelength. The motion of the filaments is then given by the Moore \& Saffman (1972) cutoff approximation to the Biot-Savart law. The configurations examined are the single infinite vortex row, the Kármán vortex street of staggered vortices, and the symmetric vortex street. These model the mixing layer, the wake, and the boundary layer, respectively. The single row was found to be most unstable to a two-dimensional disturbance, whereas the Kármán vortex street is most unstable to a three-dimensional disturbance over a significant range of street-spacing ratios. The symmetric vortex street is 
found to be most unstable to three-dimensional or two-dimensional symmetric disturbances, depending on the spacing ratio of the street. The results indicate that the mixing layer model is most unstable to a two-dimensional pairing mode, and this is the type of structure most likely to be seen. The symmetric double-layer results suggest that the coherent structures in a boundary layer will be strongly three-dimensional, which is in rough agreement with observation, whereas for the wake, the tendency to be two- or three-dimensional depends on the spacing ratio. Quantitative predictions can be made for the spreading angle of a turbulent mixing layer based on the two-dimensional dynamics of a single-vortex array of finite cross section, and they are in reasonable agreement with experiments (Moore \& Saffman 1975).

In the single-vortex ensembles discussed in Section 3, no self-consistent dynamical interaction is present between individual structures-Hill's spherical vortices or Burgers' vortices-and so no global dynamics are present. The vorticity in the structures is simplified to an extent that allows analysis of internal but strictly local vorticity dynamics, with a correspondingly local domain of validity. The kinematic models of Section 4 contain no vortex dynamics. Numerical computation of vortex interactions offers an alternative approach, and although a detailed account of numerical vortex methods lies outside the scope of this review, attempts to simulate inertial-range turbulence by the computed interaction of finite-core-sized slender vortex filaments should be highlighted. These have often focused on initial-value calculations of several interacting vortex rings. The shell-summed spectrum of a single ring of circulation $\Gamma$, radius $R$, and zero core size is given by

$$
\tilde{E}(k)=\frac{\Gamma^{2} R^{2}}{2} \int_{-1}^{1} J_{1}^{2}\left(k R \sqrt{1-\mu^{2}}\right) d \mu,
$$

where $\tilde{E}(k)$ is defined such that $\int_{0}^{\infty} \tilde{E}(k) d k$ gives the energy per unit mass integrated over all space (Leonard 1985). When $k R \gg 1, \tilde{E}$ is asymptotic to the tube-like spectrum $E(k) \sim \Gamma^{2} R /(2 k)$. The energy integral is therefore infinite, which is consistent with zero core radius. Leonard (1985) also describes calculations of five interacting vortex filaments, each of finite core radius. The filament center lines were initially of elliptical shape and positioned and oriented randomly in space. The evolution of the filaments was calculated by a vortexnumerical method, and the spectrum after some time was compared to the experimental spectra for low Reynolds number grid turbulence of Comte-Bellot \& Corrsin (1971) at the same ratio, equal to 5, of the integral scale to the Taylor microscale. The agreement is good. Follow-up calculations using a similar method and various initial geometries of vortex rings were done by Kiya \& Ishii (1991). Their evolving spectra show a $k^{-5 / 3}$ spectrum at one late time during the computation. Because this occurs over a range $k R \ll R / \sigma$, 
where $\sigma$ is the constant vortex core radius, one can infer that it arises from dynamical/numerical interaction of the rings. At this time, close approach of nearby portions of the rings is accompanied by entanglement and oscillation of the filaments. The accuracy of this type of calculation is uncertain under these circumstances. To date, no estimates of fine-scale properties have been reported using dynamical filament models.

\section{The Lundgren Stretched-Spiral Vortex}

We now return to a discussion of single-vortex models. This account of the stretched-spiral vortex model proposed by Lundgren (1982) complements and extends an earlier review by Pullin \& Saffman (1995). Approximate solutions to the unsteady Navier-Stokes equations corresponding to strictly twodimensional spiral vortices have been known since the 1930s and have found application to the structure of the starting vortex (see Saffman 1992, chapter 8) and the laminar trailing vortex (Moore \& Saffman 1973). Lundgren (1982) adapted this structure to model the fine scales of turbulence, replacing the steady Burgers' vortices in the Townsend ensemble described in Section 2 by unsteady stretched-spiral vortices. The dual properties of axial straining combined with a nonaxisymmetric vorticity structure give the model a rich predictive capability.

In polar coordinates $(s, \theta)$ in the vortex cross section, Lundgren's asymptotic solution of the vorticity equation may be expressed as

$$
\begin{aligned}
\omega(s, \theta, t) & =e^{a t} \hat{\omega}(\rho, \theta, \tau) ; \\
\hat{\omega}(\rho, \theta, \tau) & =\sum_{-\infty}^{\infty} \hat{\omega}_{n}(\rho, \tau) \exp (i n \theta) ; \\
\hat{\omega}_{n}(\rho, \tau) & =f_{n}(\rho) \exp \left[-i n \Omega(\rho) \tau-v n^{2} \Lambda^{2}(\rho) \tau^{3} / 3\right], \quad n \neq 0 ; \\
\hat{\omega}_{0}(\rho, \tau) & =g(\rho, \tau)+f_{0}(\rho, \tau) ; \\
\rho(s, t) & =s e^{a t / 2}, \quad \tau(t)=\left(e^{a t}-1\right) / a,
\end{aligned}
$$

where

$$
\begin{aligned}
\frac{1}{\rho} \frac{d}{d \rho}\left[\rho^{2} \Omega(\rho)\right] & =g(\rho)+f_{0}(\rho), \quad \text { and } \\
\Lambda & =\frac{d \Omega}{d \rho} .
\end{aligned}
$$

In Equations 8-14, $(\rho, \tau)$ are stretched space and time variables, respectively, corresponding to a strictly two-dimensional evolution (i.e. no vortex stretching $), \exp (a t) f_{0}(\rho)$ is the $\theta$-averaged vorticity for the vortex, and $\exp (a t) g(\rho)$ 
specifies some axisymmetric background vorticity field. The quantity $\Omega$ is the $\theta$-averaged angular velocity of a fluid particle for the two-dimensional motion. Equation 10 is the leading term of an asymptotic expansion in powers of $\tau^{-1}$. An arbitrary set of amplitudes $f_{n}(\rho)$ correspond to an infinite number of conserved moments of the vorticity field for inviscid two-dimensional motion. There is singular behavior at $\tau=0$. The inviscid asymptote of Equation 10 has the property that the phase of each Fourier component is constant along a common curve $\theta=\Omega(\rho) \tau$, which is of spiral form if $\Omega(\rho)$ is a monotone decreasing function of $\rho$. This gives the spiral property.

The presence of axisymmetric strain that exponentially stretches vortex lines is a crucial model ingredient. The concomitant radial compression of the vortex decreases the distance between turns of the curve $\theta=\Omega(\rho) \tau$ at a rate proportional to $t^{-3 / 2}$, compared to $\tau^{-1}$ for the equivalent unstretched vortex, thus introducing inverse one-third powers into the model. Viscosity limits the exponential stretching and smooths the radial oscillations in vorticity. The spiral structure then decays on a time scale determined by the intersection of the collapsing outer boundary of the vortex with an expanding viscous subcore region so that the vortex tends toward axisymmetry. If the total circulation is nonzero, the long-time asymptotic state is the Burgers' vortex, a result that has been all but ignored in the literature discussion of "vortex tubes."

Lundgren calculates the energy spectrum of the stretched-spiral vortex. Because the spiral is unsteady, an ansatz is required that distinguishes the instantaneous spectrum from that of an ensemble. Following Townsend (1951), this is constructed by first supposing that a box of side $L$ is populated at some absolute time $t_{a}$ by a collection of, say, $M$ vortices. The structures all undergo an identical evolution, each with a shifted time origin set at their individual instants of creation, when their initial length is $l_{0}$. At $t_{a}$, each one has an internal state corresponding to a different time instant, relative to creation, in the common evolution process. Structures are created at a rate $N_{c}$ per unit time by a process outside the model, such as the instability of larger structures or the rollup of sheets into tubes. The global vorticity distribution at $t_{a}$ is then the $\operatorname{sum} \sum_{m=1}^{M}$ of the individual vorticities. This comprises the turbulence. Vortex reconnection and/or regions near the ends of the structure axes, where vortex lines that remain parallel within individual vortices may diverge and become part of a weak background field, are ignored. A statistical equilibrium exists whereby vortices are created, stretched by the strain provided by larger scales, and then destroyed. The finite spiral lifetime conditioned by the internal dynamics was discussed above. Where the $n=0$ component of the vorticity survives the spiral, then in order to maintain equilibrium with a constant number of structures populating the box, it must be supposed that this axisymmetric fossil is destroyed by processes external to the model such as vortex breakdown or instability. This 
scenario seems natural from a physical standpoint, describing a dual tube-sheet vorticity distribution if the spiral is of the rolled-up shear layer form. Lundgren introduces an ergodic hypothesis of the form

$$
\sum_{m=1}^{M}[\ldots]=N_{c} \int_{t_{1}}^{t_{2}}[\ldots] d t,
$$

where $[\ldots]$ is any property of the spiral velocity or vorticity, $t_{1}$ is the instant of creation, and $t_{2}$ is that of destruction of the structure. This says that the ensemble average equals the time average of an individual structure over its lifetime. The assumption that the vortices are identical in evolution can be relaxed.

Applying the result $E(k)=E_{\omega \omega}(k) / 2 k^{2}$ following calculation of the vorticity spectrum $E_{\omega \omega}(k)$, Lundgren calculates the shell-summed energy (velocity) spectrum of the ensemble as

$$
\begin{aligned}
E(k)= & E_{0}(k)+\frac{4 \pi}{3} N a^{1 / 3} k^{-5 / 3} \exp \left[-\frac{2 v k^{2}}{3 a}\right] \\
& \times \sum_{n=1}^{\infty} n^{-4 / 3} \int_{0}^{\infty} \frac{\left|f_{n}(\rho)\right|^{2} \rho d \rho}{|\Lambda(\rho)|^{4 / 3}},
\end{aligned}
$$

where $N=N_{c} l_{0} / L^{3}, E_{0}(k)$ is the spectral component of the $n=0$ term in Equation 10, and the second term on the right side comes from the nonaxisymmetry. If the second term dominates $E_{0}$ at small wavenumbers then Equation 16 has a $k^{-5 / 3}$ inertial range when $k(v / a)^{1 / 2} \ll 1$. The path from Equation 10 to Equation 16 requires several clever asymptotic approximations whose subtlety has stimulated interpretive papers (e.g. Gilbert 1993). An essential part of the analytic argument involves the phase relationship $\theta=\Omega(\rho) \tau$ discussed earlier. This, together with the vortex stretching, is the key to the appearance of $k^{1 / 3}$ powers that arise in the time integral Equation 15. Equation 16 requires both vortex kinematics and dynamics and cannot be obtained by dimensional analysis of Equations 8-14. It appears to be the only known repeatable result making a definite analytical connection between a $k^{-5 / 3}$-type spectrum and an approximate solution of the Navier-Stokes equations, and for this reason it is given prominence in this review. In the spirit of Synge \& Lin (1943), the model is of the single-vortex type with the consequence that the range of spectral validity is bounded below by wavenumbers of order $2 \pi / R$, where $R$ is the maximum lateral extent of the structure. The single-vortex approximation also implies that isotropy is not required; this is because the vorticities at two points inside a structure are always parallel. The distribution of vortex-axis orientations in the box is arbitrary (Saffman \& Pullin 1994).

The stretched-spiral vortex seems to offer some hope of constructing a robust and comprehensive model of the fine scales of turbulence. For viability, such 
a model should be capable of postdicting a range of fine-scale properties other than the energy spectrum. Lundgren (1985) applies the model to the concentration spectrum of a passive and of a chemically active scalar. First, local solutions of the appropriate reaction-diffusion equations are obtained describing the evolution of a blob of the scalar as it is advected and diffused by the spiral velocity field. Attention is focused on the viscous-convective range at large Schmidt numbers, where a $k^{-1}$ spectrum $^{2}$ is found to be in agreement with a result based on scaling arguments from Batchelor (1959). The spectrum of a fast chemical reactant was found to have a substantial bump near the Batchelor wavenumber, past which the spectral decay is exponential. The spectrum in the inertial-diffusive range for the model remains an important open question.

Pullin \& Saffman (1993) have attempted to construct a standard model based on a specific realization of the spiral. They choose specific power-law forms $f_{n}(\rho)=f(\rho)$ for all $n$ and $g(\rho)$ motivated by shear-layer rollup in the presence of smooth background vorticity with zero total circulation. It is helpful to think of $f(\rho)$ and $g(\rho)$ as functions analogous to the specification of a mean velocity profile in boundary-layer calculations. The $n=0$ vorticity component is calculated from Navier-Stokes dynamics. Each vortex is assumed to have the same initial radius $R$ and circulation scale $\Gamma_{0}$, which may be interpreted as averages, over unknown statistics, of distributions of these quantities. Dimensionless parameters are defined including a production rate $N R^{2} / a$, a vortex Reynolds number $\Gamma_{0} / v$, the ratio $a R^{2} / 4 v$ of $R^{2}$ to the square of the Burgers' radius, and a parameter $a^{2} v / \bar{\epsilon}$, where $\bar{\epsilon}$ (the mean dissipation) is calculated from the model. Equations for these together with the Kolmogorov prefactor $\mathcal{K}_{0}$ and the skewness $S_{3}$ are obtained. The equations are not closed and require free parameters, chosen as $\Gamma_{0} / v$ and $N R^{2} / a$, to be fixed. Using $\Gamma_{0} / v=100$ and $N R^{2} / a=0.6$, Pullin \& Saffman (1993) solved for $a^{2} v / \bar{\epsilon}, \mathcal{K}_{0}$, and $S_{3}$ and found values of $\mathcal{K}_{0}$ and $S_{3}$ in the experimental range. In order to relate model parameters to the Taylor microscale $R_{\lambda}$, Pullin \& Saffman proposed a closure assumption $R^{2}=\lambda(4 v / a)^{1 / 2}$, where $\lambda$ is the Taylor microscale.

A reformulation of the model (Pullin et al 1994) was motivated by numerical evaluation of the spectral integrals, which showed that that the choice $\Gamma_{0} / v=$ 100 did not produce a substantial inertial range. The reformulation introduces an additional constraint, namely that the vortex structures be approximately space filling, as well as an assumption that there exists a finite upper cutoff $\tau_{2}$ that limits the growth of the core; this is chosen as the lifetime of the spiral, which can be estimated from Equation 10. The only justification given for this is that it produces a $-5 / 3$ inertial range that is not buried by a dominant core. Scaling arguments are used to relate the Taylor Reynolds number to the model

\footnotetext{
${ }^{2}$ We note here an objection to the $k^{-1}$ spectrum discussed by Dimotakis \& Miller (1990).
} 
parameters. This gives a closure that relates the model parameters, with the exception of $\Gamma_{0} / v$, to $R_{\lambda}$. A consequence is that $R=O(\lambda), L_{2}=O(\lambda)$, with weak Reynolds number dependence, where $L_{2}$ is the final stretched length of the vortex. With fixed vortex radius, the model is thus limited to scales of order $\lambda$ or smaller and so can describe only the upper inertial and dissipation ranges. There has been much past speculation on a possible interpretation of $\lambda$. Experimental measurements of zero crossings of the turbulent velocity field indicate scaling with $\lambda$ (Kailasnath $\&$ Sreenivassan 1993). This is consistent with the idea of the probe crossing the boundary region of adjacent vortex structures.

Use of this model should in principle allow the calculation of any fine-scale property of stationary turbulence. Pullin \& Saffman (1993) and Pullin et al (1994) needed to introduce statistical isotropy to compute the higher moments of the one-point velocity derivatives. Owing to divergences that result from the singular behavior of Equation 10 when $t \rightarrow 0$, the introduction of an early time cutoff in the time integration Equation 15 was required for moments higher than the third. Quantitative numerical predictions about the dependence of the one-point velocity derivative and vorticity moments on $R_{\lambda}$ and the order of the moment were in fair agreement with the experimental compilation of Van Atta \& Antonia (1980) and with the direct numerical simulation results of Vincent \& Meneguzzi $(1991,1994)$. Numerical evaluation of the time evolution of $E(k, t)$ reveals a $k^{-1}$ or tube-like range at low $k$ and a $k^{-2}$ or sheet-like range at larger $k$, with the extent of the former increasing with $t$. This confirms the sheet/tube model property discussed earlier. When numerically integrated over the spiral lifetime, the $-5 / 3$ exponent emerges from the shifting relative weights of these ranges, a result also noted by Gilbert (1993). A dissipation spectrum between an $\exp \left(-B_{1} k^{2}\right)$ and an $\exp \left(-B_{1} k\right)$ is found that suggests that the Gaussian dissipation rolloff in Equation 16 may be more a result of inaccuracies in the asymptotics than a property of the model. These results are in broad agreement with numerical calculations using the Navier-Stokes equations by Lundgren (1993).

The shell-summed spectrum of $\omega^{2}$ was estimated by Pullin \& Saffman (1994, 1995 ) and found to be in fair agreement with results from the direct numerical simulation of Jiménez et al (1993) at a moderate Reynolds number. Their analysis was generalized to higher-order powers of the vorticity by Segel (1995), for the special case when only a finite set of the $f_{n}$ s in Equations 8-10 are nonzero. The shell-summed pressure spectrum $E_{p}(k)$ was calculated by Pullin (1995), giving a leading-order nonaxisymmetric component proportional to $k^{-7 / 3}$ that is in agreement with arguments based on Kolmogorov scaling that suggest an inertial-range form $E_{p}(k)=\mathcal{K}_{p} \bar{\epsilon}^{4 / 3} k^{-5 / 3}$, where $\mathcal{K}_{p}$ is a prefactor that may be universal. Use of the standard model gives numerical values of $\mathcal{K}_{p}$ that are a factor of about two lower than those suggested by direct numerical simulation, which may also be subject to error. 
A stringent test of any model is the calculation of the longitudinal velocity structure functions

$$
B_{m}(r)=\left\langle\left(u^{\prime}-u\right)^{m}\right\rangle, \quad m=3,4, \ldots,
$$

where $u^{\prime}$ and $u$ are the velocity components at two points separated by a distance $r$, in the direction of the line joining the points. Segel (1995) claims to show that the $B_{m}(r)$ for the spiral vortex conform to the Kolmogorov scaling $B_{m}(r) \sim r^{m / 3}$. Segel's arguments appear to be essentially dimensional rather than kinematical. No dimensional prefactors are calculated. Saffman \& Pullin (1996) attempt to calculate $B_{m}(r)$ directly for both the Townsend-Burgers' vortex and the standard-model realization of the stretched spiral. Under the isotropy assumption, an expression for $B_{m}(r)$ reduces to a three-dimensional integral in the case of the Burgers' vortex and a five-dimensional one for the spiral. These are evaluated numerically, with a Monte Carlo method used for the latter case. The spiral model gives reasonable agreement for $m=2$, 4 with the data of Tabeling (P Tabeling, private communication) over a range of $r / \eta$ $\left[\eta=\left(v^{3} / \epsilon\right)^{1 / 4}\right.$ is the Kolmogorov scale] extending to $r / \eta=200$ for $m=2,4$, but there are discrepencies at higher order. Results for $B_{3}$ do not show a definite linear range corresponding to the Kolmogorov result $B_{3}(r) \sim-4 \bar{\epsilon} r / 5$.

We close this section by noting that a vortex-based model proposed by Kambe (1991) contains some of the vortex-dynamical features described here and in Section 2. There is a decomposition of the velocity and vorticity fields into vortical and background components and some use of the vorticity equation in analyzing the former. The velocity-derivative probability density functions are estimated by use of the so-called mapping closure method (see Kraichnan 1990) rather than via an average over all possible orientations of the velocity/vorticity fields.

\section{Finite-Time Singularities}

A challenging and still open question is the problem of the finite-time singularity for unsteady solutions of the Euler equation. This is a problem of vortex dynamics for the cases in which the initial vorticity distribution consists of a number of vortices or, in particular, vortex filaments. A recent hard-to-understand paper, with a reasonably complete bibliography, that claims to demonstrate the existence of a singularity is presented by Bhattacharjee et al (1995). A critique of this paper and the other numerous contributions, including computation (see Kerr 1993), is beyond the scope of this review. Indeed, in view of these developments, a new review would be useful; see Majda (1986) for a discussion.

A second open question is the significance for turbulence theory of the existence of a finite-time singularity. Bhattacharjee et al (1995) also claim to have established a connection by showing that the presence of a spiral structure in the 
initial conditions leads naturally to the Lundgren spiral-vortex model described in Section 6, from which follows in particular the Kolmogorov spectrum for the energy spectrum. A finite-time singularity would suggest that the turbulence attains a state of dynamic equilibrium in a time dependent upon the large-scale structure and independent of the viscosity, e.g. the dissipation $\epsilon$ would asymptote $u^{3} / L$ in a time $O(L / u)$ where $u$ is the rms velocity component.

An alternative approach, but one that is difficult for us to understand, has been presented by Constantin et al (1995). This work appears to consider constraints imposed on a turbulent velocity field by the requirement that a singularity does not occur. However, quantitative predictions seem to be limited to estimates of scaling lengths that cannot at present be compared to either experimental or numerical simulations.

\section{Concluding Remarks}

Vortex dynamics in turbulence have produced a spectrum of models rather than a rational theoretical framework for turbulence prediction. The task of building tractable analytical models containing some measure of global dynamics is close to Batchelor's (1953) call for a general solution of the Navier-Stokes equation in closed form for an arbitrary initial spatial distribution of velocity, and this seems no closer in the 1990s than it did 40 years ago. Stretchedvortex models of the Townsend-Lundgren type contain local dynamics, can be analyzed kinematically, and are tractable mathematically, but they rest on strong assumptions, including the absence of interactions between vortices and the neglect of axial flow in the structures. These models contain free parameters and require cutoffs in the time integration for some turbulence properties. A specific vortex structure is assumed, the stability properties of which are unknown. Contact with measurable turbulence quantities has been demonstrated with mixed results. The assumed distributions can in principle be tested against the results of direct numerical simulation (DNS) and experiments.

The "worm" structures (Jiménez et al 1993) resemble Burgers' vortices but appear to play a passive role in the overall inertial and dissipation range dynamics. Vincent \& Meneguzzi (1994) report that sheet-like structures, which roll up to form vortex tubes, are present but are not dominant in their DNS results at $R_{\lambda}=150$. Lundgren \& Mansour (1996) comment that spirals are commonly seen in experimental flow visualization but appear to be harder to observe in DNS. It remains an open question as to whether Equations 8-14, which at large times give a nearly axisymmetric vorticity distribution, can describe the internal structure of the observed tubes and spirals. The most recent studies of tubes have been limited to the visual, and a quantitative study of internal structure would seem justified. The development of a technique for characterizing tube structure remains a considerable challenge to flow visualization specialists. Particleimage velocimetery may play an important role, as may the development of 
algorithms for the detection and analysis of small-scale vortex structures in direct numerical simulation (e.g. Jiménez et al 1993, Vassilicos \& Brasseur 1996).

The axisymmetric Burgers' vortex is perhaps too simple to quantitatively model the turbulent fine scales. The Lundgren stretched spiral has a richer internal structure but contains no axial flow. The development of vortex models exhibiting truly three-dimensional structure while retaining sufficient simplicity to allow the averaging necessary for tractable computation may enhance our turbulence predictive capability as well as our understanding of the fine-scale dynamics. One possible candidate is the rectilinear vortex embedded in a linear background field of the form $u_{i}=A_{i j}(t) x_{j}, A_{i i}(t)=0$, where $x_{1}, x_{2}, x_{3}$ are laboratory-fixed coordinates. If, at some initial time, the three components of the vorticity field of the vortex alone are independent of a coordinate along the axis of the vortex, say $x_{3}^{\prime}$, but are functions of the two normal coordinates, $x_{1}^{\prime}$ and $x_{2}^{\prime}$, then the vorticity equation may be used to show that this property of independence of $x_{3}^{\prime}$ is retained in the subsequent coupled evolution of the vorticity of the vortex and of the linear field, provided that the vortex axis rotates according to

$$
\frac{\partial e_{i}}{\partial t}=e_{j} A_{i j}-e_{i} e_{k} e_{j} A_{k j}
$$

(Misra \& Pullin 1997), where $e_{i}$ are the direction cosines of the vortex axis.

This rotation is just what would be experienced by a material line element of unit length aligned with the vortex axis resulting from the linear field alone. The internal structure of the vortex allows axial flow and interaction with the background field but is two dimensional in the sense of dependence only on the cross-sectional coordinates. The vortex axis remains rectilinear. This scenario includes both the generalized Burgers' vortex and the stretched-spiral vortex. A further special case has been analyzed by Kawahara et al (1997). They consider the diffusion of a strong line vortex embedded in a simple background shear flow, and they analyze the distortion of the vortex lines of this shear by the line vortex as it rotates and diffuses. Spiral vortex structures comprising the three components of vorticity are found that resemble the Lundgren spiral with $n=2$ Fourier modes in Equation 8. The new and potentially important features of this model are, first, the presence of axial flow near the vortex core and, second, the self-consistent vortex-interaction with the weaker background shear. There is clear potential for constructing a cascade mechanism. No turbulence calculations with this model are reported by Kawahara et al (1997).

The Lagrangian filament models appear to require significant computation to produce limited results, but they do cover a fairly wide range of scales that nevertheless excludes the dissipation range. The $k^{-5 / 3}$ spectrum of the stretched spiral vortex is a consequence of the vortex internal structure and evolution and can be valid only over a range of scales with an upper bound of the vortex 
cross-section diameter. In contrast, the spectral properties of the dynamical filament models are produced by vortex-vortex interactions on scales larger than the vortex cross-section dimension. The two points of view are not inconsistent and may be complementary, describing different but overlapping ranges of scales.

The ultimate value of the structural-vortex models, which require specific vorticity distributions and which rest on the strong assertions described above, must be recognized to be questionable. In contrast, the phenomenological models, the subject of a significant part of a recent monograph (Frisch 1995) and several reviews (e.g Nelkin 1995) need no assumptions concerning field distributions but have negligible dynamical and kinematical content and hardly any connection with fluid dynamics. They contain an infinite number of free parameters, and their main contribution to our understanding of turbulence has been the postdiction of scaling exponents that may be nonexistent.

Finally, we mention briefly, as a possible application of vortex dynamics, attempts to incorporate models of large structures into turbulence prediction codes and the use of vortex structures to estimate subgrid Reynolds stresses for large eddy simulations. Reynolds \& Kassinos (1995) employ structuraleddy models for the purpose of developing a closed set of Reynolds-averaged Navier-Stokes equations. The eddies contain "vortical" and "jetal" motions and are not unlike Hill's spherical vortices. Pullin \& Saffman (1994) have suggested the use of stretched vortices for calculating subgrid stress for largeeddy simulation. This is based on a kinematic relation that gives the Reynolds stresses of an ensemble of vortices in terms of the vortex orientations and energy. They propose a specific model, which is not of the well-known Smagorinsky form, but detailed calculations for nonisotropic flows have yet to be reported.

\section{ACKNOWLEDGMENTS}

DIP was partially supported by National Science Foundation (NSF) Grant No. CTS-9634222 and PGS was partially supported by the Department of Energy under Grant No. DE-FG03-89ER25073 and by NSF Grant No. DMS-9423245.

Visit the Annual Reviews home page at http://www.AnnualReviews.org.

\section{Literature Cited}

Aref H. 1983. Integrable, chaotic, and turbulent vortex motion in two-dimensional flows. Annu. Rev. Fluid Mech. 15:345-89

Arnold VI. 1991. Kolmogorov's hydrodynamic attractors. Proc. R. Soc. London Ser. A 434: 19-22

Ashurst WT, Kerstein AR, Kerr RM, Gibson
CH. 1987. Alignment of vorticity and scalargradient in simulated Navier-Stokes turbulence. Phys. Fluids 30:2343-53

Batchelor GK. 1953. Theory of Homogeneous Turbulence. Cambridge: Cambridge Univ. Press

Batchelor GK. 1959. Small-scale variation of 
convected quantities like temperature in turbulent fluid. Part I. General discussion and the case of small conductivity. J. Fluid Mech. 5:113-33

Batchelor GK, Townsend AA. 1949. The nature of turbulent motion at large wavenumbers. Proc. R. Soc. London Ser. A 199:238-55

Bhattacharjee A, Ng CS, Wang XG. 1995. Finite-time vortex singularity and Kolmogorov spectrum in a symmetrical threedimensional spiral model. Phys. Rev. E 52: 5110-23

Brown GL, Roshko A. 1974. On density effects and large structures in turbulent mixing layers. J. Fluid Mech. 64:325-36

Buntine JD, Pullin DI. 1989. Merger and cancellation of strained vortices. J. Fluid Mech. 205:263-95

Burgers JM. 1948. A mathematical model illustrating the theory of turbulence. Adv. Appl. Mech. 1:171-199

Chorin AJ. 1980. Vortex models and boundary layer instability. SIAM J. Sci. Stat. Comput. $1: 1-21$

Chorin AJ. 1986. Turbulence and vortex stretching on a lattice. Commun. Pure Appl. Math. 39:S47-65

Chorin AJ. 1988. Vortex stretching and the small-scale structure of turbulence. Lect. N. Math. 1360:37-41

Chorin AJ. 1992. A vortex model with superfluid and turbulent percolation J. Stat. Phys. 69:67-78

Coles D. 1956. The law of the wake in turbulent boundary layers. J. Fluid Mech. 1:191-226

Comte-Bellot G, Corrsin S. 1971. Simple Eulerian time correlation of full and narrow-band velocity signals in grid generated "isotropic" turbulence. J. Fluid Mech. 48:273-37

Constantin P, Procaccia I, Segel D. 1995. Creation and dynamics of vortex tubes in three-dimensional turbulence. Phys. Rev. E 51:3207-22

Corrsin S. 1962. Turbulent dissipation fluctuations. Phys. Fluids 5:1301-2

Dimotakis PE, Miller PL. 1990. Some consequences of the boundedness of scalar functions. Phys. Fluids A 2:1919-20

Frenkiel FN, Klebanoff PS. 1971. Statistical properties of velocity differences in a turbulent field. J. Fluid Mech. 48:183-208

Frisch U. 1995. Turbulence: The Legacy of A. N. Kolmogorov. Cambridge: Cambridge Univ. Press

Gilbert AD. 1993. A cascade interpretation of the Lundgren stretched-spiral vortex model for turbulent fine structure Phys. Fluids A 5:2831-34

Jeffreys H, Jeffreys B. 1950. Methods of mathematical physics. Cambridge: Cambridge Univ. Press. 3rd ed.
Jeong G, Hussain F. 1995. On the identification of a vortex. J. Fluid Mech. 285:69-94

Jiménez J, Wray A, Saffman PG, Rogallo RS. 1993. The structure of intense vorticity in homogeneous isotropic turbulence. J. Fluid Mech. 255:65-90

Kailasnath P, Sreenivasan KR. 1993. Zero crossings of velocity fluctuations in turbulent boundary layers. Phys. Fluids A 5:2879-85

Kambe T. 1991. Cascade in turbulence and nearexponential distributions: a dynamical theory. Fluid Dyn. Res. 8:159-73

Kawahara G, Kida S, Tanaka M, Yanase S. 1997. Wrap, tilt and stretch of vorticity lines around a strong straight vortex tube in a simple shear flow. J. Fluid Mech. In press

Kerr RM. 1985. Higher-order derivative correlations and the alignment of small-scale structures in isotropic numerical turbulence. J. Fluid Mech. 153:31-58. See also NASA TM (84407), 1983.

Kerr RM. 1990. Velocity scalar and transfer spectra in numerical turbulence. J. Fluid Mech. 211:309-22

Kerr RM. 1993. Evidence for a singularity of the 3-dimensional, incompressible Euler equations. Phys. Fluids A 7:1725-46

Kida S, Murakami Y. 1987. Kolmogorov similarity in freely decaying turbulence. Phys. Fluids 30:2030-39

Kiya M, Ishii H. 1991. Vortex interaction and Kolmogorov spectrum. Fluid Dyn. Res. 8:7383

Kraichnan RH. 1959. The structure of isotropic turbulence at very high Reynolds numbers. $J$. Fluid Mech. 5:497-53

Kraichnan RH. 1990. Models of intermittency in hydrodynamic turbulence. Phys. Rev. Lett. 65:575-78

Kuo AY, Corrsin S. 1972. Experiment on the geometry of the fine-structure regions in fully turbulent fluid. J. Fluid Mech. 56:447-79

Landau LD, Lifshitz EM. 1959. Fluid Mechanics. London/New York: Pergamon

Leonard A. 1980. Vortex methods for flow simulation. J. Comp. Phys. 37:289-335

Leonard A. 1985. Computing three-dimensional incompressible flows with vortex elements. Annu. Rev. Fluid Mech. 17:52359

Lundgren TS. 1982. Strained spiral vortex model for turbulent fine structure. Phys. Fluids 25:2193-203

Lundgren TS. 1985. The concentration spectrum of the product of a fast biomolecular reaction. Chem. Eng. Sci. 40:1641-52

Lundgren TS. 1993. A small scale turbulence model. Phys. Fluids A 5:1472-83

Lundgren TS, Mansour NN. 1996. Transition to turbulence in an elliptic vortex. J. Fluid Mech. 307:43-62 
Majda A. 1986. Vorticity and the mathematical theory of incompressible fluid flow. Commun. Pure Appl. Math. 39:S187-220

Meiburg E. 1995. Three-dimensional vortex dynamics simulations. In Fluid Vortices, ed. SI Green, pp. 651-79. Dordrecht: Klewer Acad.

Melander MV, Hussain F. 1994. Topological vortex dynamics in axisymmetrical viscous flows. J. Fluid Mech. 260:57-80

Meshalkin LD, Sinai YG. 1961. Investigation of the stability of a stationary solution of a system of equations for the plane movement of an incompressible viscous liquid. Appl. Math. Mech. 30:1700-5

Min IA, Mezić I, Leonard A. 1996. Lévy stable distributions for velocity and velocity difference in systems of vortex elements. Phys. Fluids 8:1169-80

Misra A, Pullin DI. 1997. A vortex-based subgrid model for large-eddy simulation. Phys. Fluids. In press

Moffatt HK, Kida S, Ohkitani K. 1994. Stretched vortices - the sinews of turbulencelarge Reynolds number asymptotics. J. Fluid Mech 259:241-64

Moore DW, Saffman PG. 1972. The motion of a vortex filament with axial flow. Phil. Trans. R. Soc. London Ser. A 272:403-29

Moore DW, Saffman PG. 1973. Axial flow in laminar trailing vortices. Proc. R. Soc. London Ser. A 333:491-508

Moore DW, Saffman PG. 1975. The density of organized vortices in a turbulent mixing layer. J. Fluid Mech. 69:465-73

Nelkin M. 1994. Universality and scaling in fully-developed turbulence. Adv. Phys. 43:143-81

Perry AE, Chong MS. 1982. On the mechanism of wall turbulence. J. Fluid Mech. 119:173217

Perry AE, Marušić I. 1995. A wall-wake model for the turbulence structure of boundary layers. Part I. Extension of the attached eddy hypothesis. J. Fluid Mech. 298:361-88

Porter DH, Pouquet A, Woodward PR. 1994. Kolmogorov-like spectra in decaying three-dimensional turbulence. Phys. Fluids 6:2133-42

Pullin DI. 1995. Pressure spectra for vortex models of homogeneous turbulence. Phys. Fluids 7:849-56

Pullin DI, Buntine JD, Saffman PG. 1994. On the spectrum of a stretched spiral vortex. Phys. Fluids 6:3010-27

Pullin DI, Saffman PG. 1993. On the LundgrenTownsend model of turbulent fine scales. Phys. Fluids A 5:126-45

Pullin DI, Saffman PG. 1994. Reynolds stresses and one-dimensional spectra for vortex models of homogeneous anisotropic turbulence. Phys. Fluids 6:1787-96
Pullin DI, Saffman PG. 1995. Vortex-models of the fine-scales of turbulence. In SmallScale Structuresin Three-Dimensional Hydrodynamic and Magnetohydrodynamic Turbulence, ed. M Meguzzi, A Pouquet, P-L Sulem, pp. 61-74. New York: SpringerVerlag

Reynolds WC, Kassinos S. 1995. One-point modeling of rapidly deformed homogeneous turbulence. Proc. R. Soc. London Ser. A 451: 87-104

Robinson AC, Saffman PG. 1982. Threedimensional stability of vortex arrays. $J$. Fluid Mech. 125:411-27

Robinson AC, Saffman PG. 1984. Stability and structure of stretched vortices. Stud. Appl. Math. 70:163-81

Saddoughi SG, Veeravalli SV. 1994. Local isotropy in turbulent boundary-layers at high Reynolds numbers. J. Fluid Mech. 268:33372

Saffman PG. 1967. The large-scale structure of homogeneous turbulence. J. Fluid Mech. 27:581-93

Saffman PG. 1970. Dependence on Reynolds number of higher-order moments of velocity derivatives in isotropic turbulence. Phys. Fluids 13:2193-94

Saffman PG. 1992. Vortex Dynamics. Cambridge: Cambridge Univ. Press

Saffman PG, Baker GR. 1979. Vortex interactions. Annu. Rev. Fluid Mech. 11:95-122

Saffman PG, Pullin DI. 1994. Anisotropy of the Lundgren-Townsend model of fine-scale turbulence. Phys. Fluids 6:802-7

Saffman PG, Pullin DI. 1996. Calculation of velocity structure functions for vortex models of isotropic turbulence. Phys. Fluids 8:307284

Segel D. 1995. The higher moments in the Lundgren model conform with Kolmogorov scaling. Phys. Fluids 7:3072-77

Skåre PE, Krogstad PA. 1994. A turbulent equilibrium boundary layer near separation. $J$. Fluid Mech. 272:319-48

Synge JL, Lin CC. 1943. On a statistical model of isotropic turbulence. Trans. R. Soc. Canada 37:45-79

Taylor GI. 1915. Eddy motion in the atmosphere. Philos. Trans. R. Soc. London Ser. A 215:1-26

Taylor GI. 1932. The transport of vorticity and heat through fluids in turbulent motion. Proc. R. Soc. London Ser. A 135:685-705

Taylor GI. 1935. Distribution of velocity and temperature between concentric rotating cylinders. Proc. R. Soc. London Ser. A 151: 494-512

Taylor GI. 1937. Flow in pipes and between parallel planes. Proc. R. Soc. London Ser. A 159:496-506 
Taylor GI, Green AE. 1937. Mechanism of the production of small eddies from large ones. Proc. R. Soc. London Ser. A 158:499-521

Tennekes AA. 1968. Simple model for the small-scale structure of turbulence. Phys. Fluids 11:669-70

Townsend AA. 1951. On the fine-scale structure of turbulence. Proc. R. Soc. London Ser. A 208:534-42

Townsend AA. 1976. The Structure of Turbulent Shear Flow. Cambridge: Cambridge Univ. Press

Townsend AA. 1987. Organized eddy structures in turbulent flows. Physicochem. Hydrodyn. 8:23-30

Van Atta CW, Antonia RA. 1980. Reynolds number dependence of skewness and flatness factors of turbulent velocity derivatives. Phys. Fluids 23:252-57

Vassilicos JC, Brasseur JG. 1996. Self-similar spiral flow structure in low-Reynolds number isotropic and decaying turbulence. Phys. Rev. E 54:467-85

Vincent A, Meneguzzi M. 1991. The spatial structure and statistical properties of homogeneous turbulence. J. Fluid Mech. 225:1-20

Vincent A, Meneguzzi M. 1994. The dynamics of vorticity tubes in homogeneous turbulence. J. Fluid Mech. 258:245-54

von Kármán T, Howarth L. 1938. On the statistical theory of isotropic turbulence. Proc. $R$. Soc. London Ser. A 164:192-215 


\section{CONTENTS}

Lewis Fry Richardson and His Contributions to Mathematics,

Meteorology, and Models of Conflict, J.C.R. Hunt

Aircraft Laminar Flow Control , Ronald D. Joslin 1

Vortex Dynamics in Turbulence, D. I. Pullin, P. G. Saffman 31

Interaction Between Porous Media and Wave Motion, A. T. Chwang, A. T. Chan

Drop and Spray Formation from a Liquid Jet, S. P. Lin, R. D. Reitz 85

Airplane Trailing Vortices, Philippe R. Spalart 107

Diffuse-Interface Methods in Fluid Mechanics, D. M. Anderson, G. B. 139

McFadden, A. A. Wheeler

Turbulence in Astrophysics: Stars, V. M. Canuto, J. ChristensenDalsgaard

Vortex-Body Interactions, Donald Rockwell

Nonintrusive Measurements for High-Speed, Supersonic, and Hypersonic Flows, J. P. Bonnet, D. Grésillon, J. P. Taran

Renormalization-Group Analysis of Turbulence, Leslie M. Smith, Stephen L. Woodruff

Control of Turbulence, John Lumley, Peter Blossey

Direct Simulation Monte Carlo--Recent Advances and Applications, E.S. Oran, C.K. Oh, B.Z. Cybyk

Computational Hypersonic Rarefied Flows, M. S. Ivanov, S. F. Gimelshein

Turbulent Flow Over Hills and Waves, S. E. Belcher, J. C. R. Hunt 507

Direct Numerical Simulation: A Tool in Turbulence Research, Parviz 539

Moin, Krishnan Mahesh

Micro-Electro-Mechanical-Systems (MEMS) and Fluid Flows, ChihMing Ho, Yu-Chong Tai 